\title{
Basmati Rice Fraud under the Magnifying Glass of DNA Analysis
}

\author{
Françoise Fridez
}

\begin{abstract}
Basmati rice is a perfumed rice of high commercial value which is easily adulterated. A list of rice varieties accepted as true Basmati is the subject of an agreement between Europe, Pakistan and India. Each rice variety can be characterized by DNA profiling, thus offering to the control authorities a powerful tool to detect fraud. Mixtures of authentic Basmati and non-Basmati varieties can be quantified. During the last six years, about one third of the analysed samples proved to be fraudulent.
\end{abstract}

Keywords: Basmati rice $\cdot$ DNA profiling $\cdot$ Fraud $\cdot$ Microsatellite $\cdot$ STR

\section{Introduction}

Basmati rice is one of the finest types of rice. It is a perfumed rice of high commercial value, which tempts the avarice of companies and leads some of them to fraud. Its price mirrors these qualities as it sells for two to three times the price of other long grain rice. It is indeed recognized for its distinctive aroma and for its unique physical, cooking and taste properties. Its grains are typically thin and their length almost double during cooking without suffering from agglutination because of its high amylopectin and amylose content.

Basmati is the customary name for certain varieties of rice with these unique properties that are grown exclusively in specific areas of the Indo Gangetic Plains. It is thus grown only in India and Pakistan. But world demand has exploded during the last decade. It is thus not a surprise that we find Basmati rice mixed with various proportions of lower quality varieties on the world markets. However, fighting fraud has to rely on a clear definition of what is authentic Basmati and what is not.

${ }^{*}$ Correspondence: F. Fridez, PhD

Service de la consommation et des affaires vétérinaires

rue Jehanne-de-Hochberg 5

$\mathrm{CH}-2000$ Neuchâtel

E-mail: Francoise.Fridez@ne.ch
This definition was agreed upon by the countries concerned and their European commercial partner countries. This led to the European regulations EC 1549/2004 and EC 1234/2007,[1] which contain a list of the nine approved Basmati rice varieties (Basmati 370, Type-3 (Dehradun), Basmati 217, Ranbir Basmati, Taraori Basmati (HBC-19), Basmati 386, Kernel (Basmati), Super Basmati, Pusa Basmati). Moreover, the rice industry organizations and state regulatory services from India, Pakistan and United Kingdom have agreed on a 'Code of practice on Basmati rice', ,2] which approved six further varieties (Basmati 198, Basmati 385, Kasturi (IET 8580), Haryana Basmati (HKR 228/IET 10367), Mahi Suganda, Punjab Basmati (Bauni Basmati)). It should be emphasized that the boundaries of the definition of Basmati are somewhat arbitrary. The needs for regulation in a global economy are not adapted to the local Asian economy where millions of rice-producing farmers have developed an unknown number of varieties, which could not all be taken into account during the design of the regulations.

The Code of practice on Basmati rice lists almost 20 examples of rice varieties which are not approved as Basmati rice and which might be used to fake Basmati rice. But the list of potential fraudulent varieties is naturally longer. Throughout six years of our Basmati rice analyses, more than 150 different non-Basmati varieties have been detected through the analysis of 176 supposed Basmati samples! The rice industry organizations and the regulatory services from India, Pakistan and United Kingdom have agreed on a tolerance threshold for the presence of non-Basmati rice: The maximum limit for non-basmati rice is $7 \%$, which has important consequences for the laboratories. In the absence of specific regulations in Switzerland, we use the British code of practice and the European regula- tion as our legal reference documents. We thus consider their combined list of 15 different Basmati rice varieties as authentic. This is also the current practice across all Europe.

\section{Analytical Strategy}

DNA analysis is the appropriate method to determine if Basmati rice is authentic, faked or a mixture with authentic and non-approved varieties. Its goal is also to measure the proportions of the mixtures and to determine if the non-approved varieties are present beyond the 7\% threshold.

DNA, from rice or a human being or any living being, is a long molecule made of coding regions which correspond to the genes interspersed with long non coding stretches. In these stretches, DNA displays tandem repeats, like the stutters in the human language. These are repetitive sequences usually called STR (for Short Tandem Repeats), sometimes also called microsatellites. They are also called DNA markers because the number of repetitions may be very different from one variety to the other. It is those differences which allow the identification of the Basmati rice varieties, exactly as human identification in forensic science (Fig. 1). We use a set of seven different DNA markers to test the rice. ${ }^{[3]}$

For the analysis, a representative part of the sample is first ground into a fine powder and mixed in a solution able to dissolve all the biological molecules. DNA is then captured on a special DNA binding filter, while the other molecules are washed away. DNA can then be recovered, pure, by using a special DNA recovery solution.

A kind of molecular copying process is then used, which replicates a million times the specific STRs of interest. This process is called PCR (polymerase chain reaction). 


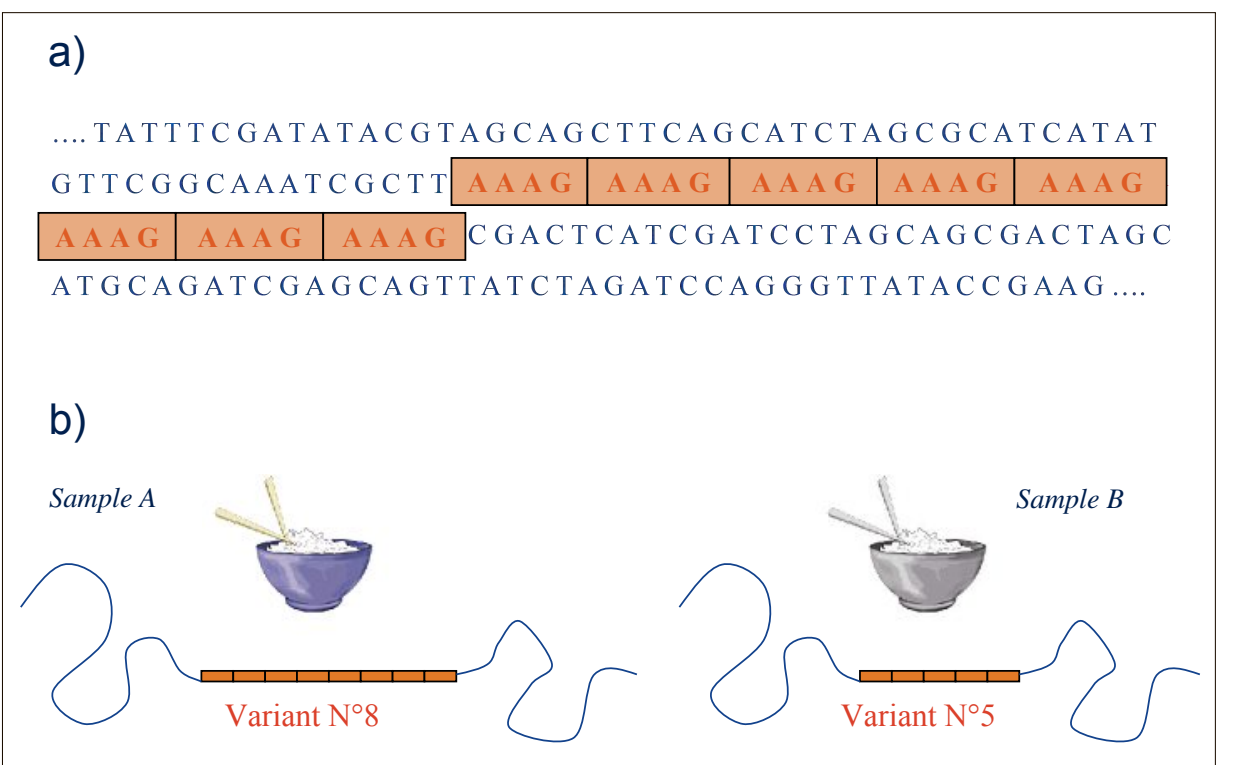

and the other is not, it is even possible to make quantitative inferences by using peak height ratios for the two varieties present in the mixture. It can thus be determined if the non-Basmati has a proportion higher than the legal $7 \%$ allowance.

Most cases are however more complex, with three or more varieties present. In such cases, the only way to unequivocally identify the varieties in the mixtures without making spurious assumptions is to analyse individual grains, each providing single DNA profiles easy to interpret. Indeed there are no length variants specific to the true Basmati varieties. What is specific for a variety is only the set of variants on several markers. The interpretation then relies on the counting of the Basmati and non-Basmati individual grains to get the

Fig. 1. a) Stretch of rice DNA sequence containing an AAAG repetitive sequence, whose elements are highlighted by rectangles. b) The number of repetitions may be different from one rice variety to the other, thus providing a basis for variety identification.

It offers us millions of copies of exactly the part of DNA we want to look into details during the next step of the DNA analysis.

The replicated DNA sequences are then separated in a capillary electrophoresis instrument according to their size or their length: driven by a powerful electric field, the DNA fragments move through the capillary at a speed inversely proportional to their size. The short fragments reach the detector at the other end of the capillary before the larger fragments. The detection profile offers the image of peaks corresponding to the fragments present in the sample (Fig. 2). When comparing with appropriate electrophoresis standards, it is possible to identify the repetitive sequence variants present in the sample. Both the PCR and the electrophoresis can be used for the simultaneous analysis of several STRs at the same time (multiplex analysis). The set of variants expected for each DNA marker for the authentic Basmati varieties is naturally known (Fig. 3).

\section{Results and Interpretation}

In simple cases (one single rice variety present in the sample), the interpretation is straightforward by simply checking if the set of variants detected corresponds to a true Basmati. When two varieties are present in a sample, it may be possible to infer the set of variants owned by the two varieties by looking at the peak heights in the DNA profile, and then compare with what is expected from true Basmati for each of them. When one variety is a true Basmati

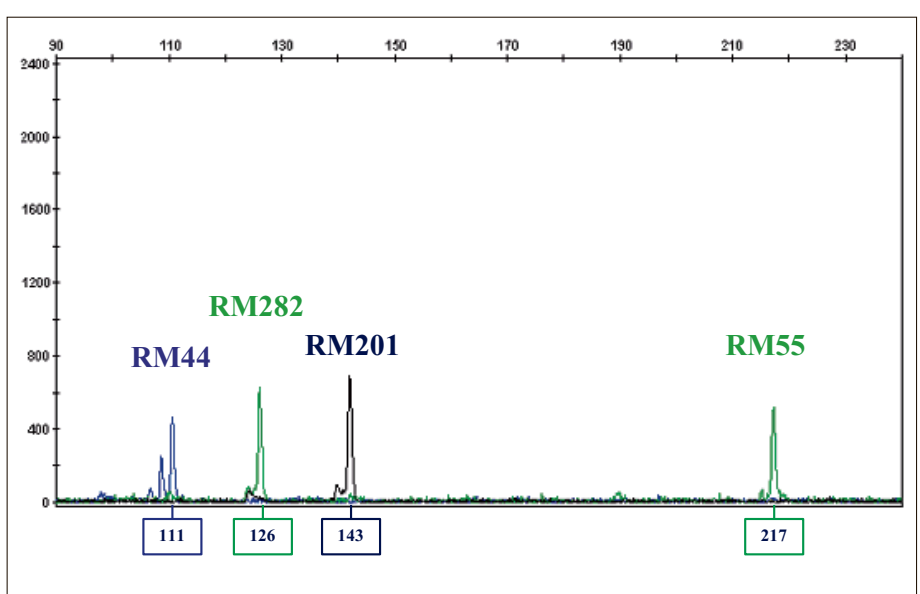

Fig. 2. Example of a DNA profile obtained through the simultaneous analysis of four STRs (multiplex analysis). In this example, the sample contains grains which all belong to the same Basmati variety: Taraori Basmati. A single peak is thus obtained for each marker analysed (for the marker RM44, the other peaks are stutters).

\begin{tabular}{|l|c|c|c|c|c|c|c|}
\hline & RM212 & RM282 & RM263 & RM44 & RM201 & RM339 & RM55 \\
\hline Basmati 370 group & 117 & 126 & 155 & 107 & 143 & 181 & 233 \\
\hline Dehra Dun (Type3) & 117 & 126 & 160 & 107 & 143 & 181 & 233 \\
\hline Basmati 217 & 117 & 126 & 155 & 107 & 143 & 181 & 233 \\
\hline Ranbir Basmati & 117 & 126 & 155 & 107 & 143 & 181 & 233 \\
\hline Taraori & 117 & 126 & 160 & 111 & 143 & 181 & 217 \\
\hline Basmati 386 & 117 & 126 & 160 & 111 & 143 & 181 & 217 \\
\hline Kernel & 117 & 126 & 160 & 111 & 143 & 181 & 217 \\
\hline Super Basmati & 117 & 126 & 160 & 107 & 143 & 184 & 217 \\
\hline Pusa Basmati & 117 & 134 & 160 & 111 & 143 & 181 & 228 \\
\hline Basmati 198 & 135 & 126 & 155 & 107 & 143 & 181 & 233 \\
\hline Basmati 385 & 135 & 126 & 160 & 111 & 143 & 181 & 233 \\
\hline Kasturi & 115 & 126 & & 101 & 143 & 145 & 228 \\
\hline Haryana Basmati & 135 & 126 & 155 & 101 & 143 & 145 & 228 \\
\hline Mahi Sugandha & 135 & 132 & 155 & 101 & 158 & 145 & 228 \\
\hline Punjab Basmati & 135 & 132 & 155 & 107 & 143 & 181 & 233 \\
\hline Basmati 2000 & 135 & 126 & 160 & 111 & 143 & 184 & 217 \\
\hline Shaheen Basmati & 135 & 134 & 160 & 111 & 143 & 181 & 217 \\
\hline Sherbati short & 113 & 132 & 180 & 101 & 160 & 145 & 228 \\
\hline Sherbati & 113 & & 180 & 101 & 160 & 145 & 228 \\
\hline Sherbati aw ns & 113 & 132 & 180 & 101 & 160 & 145 & 228 \\
\hline Mugad Sugandha & 115 & 134 & 180 & 101 & 160 & 145 & 228 \\
\hline Pak 386 & 113 & 132 & & 101 & 160 & 145 & 228 \\
\hline Superfine & 115 & 134 & 180 & 111 & 160 & 145 & 228 \\
\hline Pusa Sugandha & 115 & 134 & 160 & 101 & 143 & 158 & 228 \\
\hline Pusa Sugandha 2 & 115 & 134 & 160 & 101 & 143 & 158 & 228 \\
\hline Pusa Sugandha 3 & 115 & 134 & 160 & 101 & 143 & 158 & 228 \\
\hline Yamini & 117 & 126 & 160 & 107 & 143 & 181 & 217 \\
\hline
\end{tabular}

Fig. 3. List of the DNA variants owned by various rice varieties. The names of the authentic Basmati varieties are written in red and blue on the left column, while several non-Basmati varieties are also displayed (names in green). The head of the columns provide the names of the DNA markers analysed. The names of the variants for each DNA markers are numbers which correspond to the apparent size as estimated through capillary electrophoresis. In this figure, it is clearly visible that any size variant for a DNA marker is not specific for a single DNA variety. It is only the combination of variants across several DNA markers which is specific of a rice variety. 
estimated proportion. Bayesian statistics is then used to calculate the confidence intervals. ${ }^{[4]}$

\section{Our Experience over the Past Years and the Challenges Ahead}

For the past six years, our laboratory has controlled the authenticity of Basmatilabelled rice samples available on the Swiss market, and samples sent by consumers' organisations in France and Denmark. 176 samples have thus been analysed. 54 samples (corresponding to $31 \%$ of the total) were considered fraudulent since they contained more than $7 \%$ non-Basmati rice.

Most of the non-compliant samples contained a proportion of non-Basmati varieties between 7 and $30 \%$. But it appeared that some samples contained only a minor fraction of true Basmati or even no Basmati rice at all. Among the samples which have been considered as compliant, it is worth mentioning that $35 \%$ of them did contain some non-Basmati rice but below the tolerance threshold of $7 \%$.

It appears that some exporting producers claim that the list of approved varieties is too restrictive, and outdated. They would like other more modern and more productive varieties to be included onto the list. And indeed the DNA profiles of some of them are very close to those of some Basmati varieties of the list. They may display the same repetitive sequence variants for all DNA markers except one. When two laboratories analyse the same sample using a different set of DNA markers, it may happen that the first laboratory has analysed the only DNA marker which reveals that the variety is not a true Basmati. The marker set of the second laboratory may not include this DNA marker and thus may not be able to get this information for this specific variety. For another variety, the situation may be the reverse: the second laboratory may have the only DNA marker in its set which proves the sample to be non-Basmati, while the first laboratory does not analyse this marker. Some level of standardization of the analytical practice is thus necessary.

Moreover, some private laboratories active on the market of food control have the practice of being tolerant towards some of the above-mentioned varieties that are very close to approved ones. They are sensitive to the arguments of the exporting producers. But while doing so, they are running ahead of the future legal frame changes, but these are not yet in place. It is up to the interested parties (exporters and importers) to take the necessary steps to get this legal frame adjusted, before the exporters relax their labelling practice. In Switzerland, an official information sheet might soon be distributed to the interested parties to prevent any misunderstanding.

Basmati rice investigation is a good example of the complexity of the mission of food control agencies. First the border between a compliant and non-compliant sample is intrinsically arbitrary. Secondly, this limit is a moving one. We can expect that the European Union will adjust its regulations if India and Pakistan make the request. And finally, the enforcement of the regulations has to rely on analytical technologies able to draw a clear line at the sometimes difficult border between compliant and non-compliant samples.

Received: December 30, 2015

[1] EC 1549/2004: http://eur-lex.europa.eu/legalcontent/EN/TXT/PDF/?uri=CELEX:32004R15 49\&from=EN, C 1234/2007: http://www.wipo. int/edocs/lexdocs/laws/fr/eu/eu129fr.pdf.

[2] British Retail Consortium, July 2005, www.brc. org.uk/Downloads/Basmati_Code.pdf.

[3] K. Steele, CAZS Natural Resources, Bangor University, 22 September 2009 http://ec.europa. eu/agriculture/analysis/external/basmati/ssr markers_steele_en.pdf.

[4] A. Biedermann, F. Taroni, S. Bozza, C. G. G. Aitken, Law, Probability and Risk 2008, 7, 35. 